Exploring the risk factors for differences in the cumulative incidence of coeliac disease in two neighboring countries : the prospective DIABIMMUNE study

\title{
Simre, Kart
}

\section{6-11}

Simre , K, Uibo , O , Peet , A , Tillmann , V , Kool , P , Hämäläinen , A-M , Harkonen , T , Siljander, H , Virtanen , S , Ilonen , J , Knip , M , Uibo , R \& DIABIMMUNE Study Grp 2016 , ' Exploring the risk factors for differences in the cumulative incidence of coeliac disease in two neighboring countries : the prospective DIABIMMUNE study ', Digestive and Liver Disease , vol. 48 , no. 11 , pp. 1296-1301 . https://doi.org/10.1016/j.dld.2016.06.029

http://hdl.handle.net/10138/228786

https://doi.org/10.1016/j.dld.2016.06.029

publishedVersion

Downloaded from Helda, University of Helsinki institutional repository.

This is an electronic reprint of the original article.

This reprint may differ from the original in pagination and typographic detail.

Please cite the original version. 
Alimentary Tract

\title{
Exploring the risk factors for differences in the cumulative incidence of coeliac disease in two neighboring countries: the prospective DIABIMMUNE study
}

\author{
Kärt Simre ${ }^{\mathrm{a}, \mathrm{b}}$, Oivi Uibo ${ }^{\mathrm{b}, \mathrm{c}}$, Aleksandr Peet ${ }^{\mathrm{b}, \mathrm{c}}$, Vallo Tillmann ${ }^{\mathrm{b}, \mathrm{c}}$, Pille Kool ${ }^{\mathrm{c}}$, \\ Anu-Maaria Hämäläinen ${ }^{\mathrm{d}}$, Taina Härkönen ${ }^{\mathrm{e}, \mathrm{f}}$, Heli Siljander ${ }^{\mathrm{e}, \mathrm{f}}$, Suvi Virtanen ${ }^{\mathrm{g}, \mathrm{h}, \mathrm{i}}$, \\ Jorma Ilonen $^{\mathrm{j}}$, Mikael Knip ${ }^{\mathrm{e}, \mathrm{f}, \mathrm{i}, \mathrm{k}}$, Raivo Uibo ${ }^{\mathrm{a}, *}$, The DIABIMMUNE Study Group ${ }^{1}$ \\ a Department of Immunology, Institute of Biomedicine and Translational Medicine, University of Tartu, Tartu, Estonia \\ b Children's Clinic of Tartu University Hospital, Tartu, Estonia \\ ${ }^{\mathrm{c}}$ Department of Pediatrics, University of Tartu, Tartu, Estonia \\ d Jorvi Hospital, Helsinki University Hospital, Espoo, Finland \\ e Children's Hospital, University of Helsinki and Helsinki University Hospital, Helsinki, Finland \\ ${ }^{\mathrm{f}}$ Research Programs Unit, Diabetes and Obesity, University of Helsinki, Helsinki, Finland \\ g Nutrition Unit, Department of Lifestyle and Participation, National Institute for Health and Welfare, Helsinki, Finland \\ ${ }^{\mathrm{h}}$ School of Health Sciences, University of Tampere, Tampere, Finland \\ ${ }^{i}$ Tampere Center for Child Health Research, Tampere University Hospital, Tampere, Finland \\ j Immunogenetics Laboratory, University of Turku and Turku University Hospital, Turku, Finland \\ ${ }^{\mathrm{k}}$ Folkhälsan Research Center, Helsinki, Finland
}

\section{A R T I C L E I N F O}

\section{Article history:}

Received 15 March 2016

Accepted 23 June 2016

Available online 6 July 2016

\section{Keywords:}

Breastfeeding

Coeliac disease

Early feeding

Early infections

\begin{abstract}
A B S T R A C T
Background: During the last several decades the prevalence of coeliac disease (CD) has increased worldwide.

Aim: To compare the cumulative incidence of CD between Estonian and Finnish children and to identify the risk factors.

Materials and methods: Children were recruited as part of the DIABIMMUNE Study. In the birth cohort (BC) 258 children from Estonia and 305 from Finland, and in the young children's cohort (YCC) 1363 and 1384 children were followed up, respectively. The diagnosis of CD was made in accordance with the ESPGHAN guidelines-the presence of IgA-tTG antibodies and small bowel villous atrophy.

Results: During the study period 29 children developed CD. The cumulative incidence of CD was significantly higher in Finland $(0.77 \%$ vs $0.27 \% ; P=0.01)$. No difference was seen between the children with $C D$ and the controls in the duration of breastfeeding or the age at cereal introduction. The BC children with $\mathrm{CD}$ had had significantly more episodes of infections with fever by the age of 12 months compared to the controls (3.4 vs $1.4 ; P=0.04$ ).

Conclusion: The 5-year cumulative incidence of childhood CD is significantly higher in Finland than in Estonia. Sequential infections early in life may increase the risk for developing CD.
\end{abstract}

(C) 2016 Published by Elsevier Ltd on behalf of Editrice Gastroenterologica Italiana S.r.l.

\section{Introduction}

Coeliac disease (CD) is a dietary gluten induced immunemediated systemic disorder that occurs in genetically predisposed

\footnotetext{
${ }^{1}$ See Appendix A.

* Corresponding author at: Department of Immunology, Institute of Biomedicine and Translational Medicine, University of Tartu, 19 Ravila Street, Tartu 50411, Estonia.

E-mail address: raivo.uibo@ut.ee (R. Uibo).
}

people. It is characterized by variable clinical manifestations, CD-specific autoantibodies, HLA-DQ2 (DQA $1 * 05-D Q B 1 * 02)$ or HLADQ8 (DQB1*03:02) haplotypes and enteropathy [1].

In recent decades the prevalence of $C D$ has increased worldwide [2] and is now estimated to range between $1 \%[3,4]$ and $3 \%[5]$ in the Western countries. The frequency has also increased in Estonia and in Finland, the two neighbouring countries, with marked differences in the socio-economic background until the early 1990s. It was shown that the age-standardized incidence rate of childhood CD in Estonia increased dramatically during a 35-year period: from 0.10 in $1976-1980$ to 3.14 per 100,000 person-years in $2006-2010$ 
[6]. However, it is still lower than in some Western countries $[7,8]$. In Finland the total prevalence of $\mathrm{CD}$ has doubled during the last two decades and is as high as $2 \%$ [9].

The exact reasons why the frequency of $C D$ is increasing are unknown. The increase is partly due to the improved serological testing and awareness of $\mathrm{CD}$ but this cannot explain the overall increase. There are important genetic, environmental and immunological factors that contribute to the pathogenesis of $C D$ [10]. More than $95 \%$ of patients with CD are HLA-DQ2 positive and most of the remaining carry HLA-DQ8 $[1,11]$. The frequency of subjects positive for either of HLA-DQ2 and/or HLA-DQ8 in the general population may be as high as $40 \%$ and that of high-risk associated HLA-DQ alone more than $20 \%$ [12].

It is not known why some people with strong genetic predisposition develop $\mathrm{CD}$ whereas others do not. The reasons could be related to dietary changes and/or infections. Some previous studies have shown that exclusive or any breastfeeding, as well as breastfeeding at the time of gluten introduction, may reduce the risk of developing CD during childhood [13-15]. Some authors have reported that the time of gluten introduction may affect the risk for developing CD $[16,17]$. However, recent randomized prospective studies have challenged the idea about association between the timing of gluten introduction and the risk for $\mathrm{CD}$. Vriezinga et al. found that the introduction of small quantities of gluten at 4-6 months of age in at-risk children did not reduce the incidence of $C D$ by the age of 3 years [18]. Lionetti et al. reported that neither the delayed introduction of gluten nor breastfeeding reduced the rate of $\mathrm{CD}$ in high-risk children, whereas later introduction of gluten was associated with delayed manifestation of the clinical disease [19]. In addition, seasonal variation in the birth date of patients with $C D$ has been reported [20], indicating that infections in infancy may be involved in the pathogenesis [21]. However, the exact triggering infections and underlying principal mechanisms are not fully understood.

The aims of the current analysis were to compare the cumulative incidence of $C D$ between Estonian and Finnish children up to 5 years of age and to identify the factors that might be involved in modulating the incidence, paying special attention to early feeding and infections.

\section{Materials and methods}

\subsection{Study design and population}

Children were recruited into the study cohort as part of the international prospective observational DIABIMMUNE Study. The DIABIMMUNE Study set out to assess the role of the hygiene hypothesis in the development of type 1 diabetes (T1D) and other immune-mediated diseases. The study was carried out in Estonia, Finland and Russian Karelia from September 2008 to October 2013. The study included two cohorts: a birth cohort (BC) observed from birth up to the age of 3 years and a cohort of young children (YCC) examined for the first time at the age of 3 years and followed up to the age of 5 years.

Initially, 2714 infants from maternity hospitals in Estonia and 3105 in Finland were recruited to the BC. Cord blood samples were taken from all children wherever it has been technically possible and parents have given written informed consent. All children were analyzed for T1D/CD associated HLA DR/DQ alleles as described earlier [22]. Four hundred and twenty-six Estonian children and 713 Finnish children carried an eligible HLA genotype (DR3-DQ2 (DQA1*05-DQB1*02)/DR4DQ8 (DRB1*04:01/2/4/5-DQA1*03-DQB1*03:02) heterozygosity, or either DR4-DQ8 or DR3-DQ2 haplotype alone without any haplotypes protecting for T1D). Children with eligible HLA were invited for a follow-up study. In total, 258 children in Estonia (61\% of eligible) and 305 in Finland (43\% of eligible) completed the 3-year follow-up programme with planned visits at the age of $3,6,12$, 18,24 and 36 months ( \pm 1 month). Finns had higher frequency of HLA risk genotypes (23.0\% vs $15.7 \%$ ), but Estonians consented more eagerly (80.5\% vs 58.6\%). Both came to the 3 year visit with the same eagerness. As more Finnish children did not give the consent for follow-up and we do not have any information about them and their diseases, we cannot totally exclude that this could affect our results. Accordingly one might suppose that there are even bigger differences between Estonia and Finland. At the first study visit the parents were given a diary in which they were asked to report data about infections, use of medications, allergies, vaccinations and environmental exposures. Data on breastfeeding and on the introduction of complementary foods and their character was also registered. All diary data was transferred to a central database at each visit.

In the YCC, 1681 children from Estonia and 1575 children from Finland were studied at the age of 3 years, and $1363(81 \%)$ and 1384 (88\%) children, respectively, attended the follow-up visit at the age of 5 years. In addition, 250 children from Estonia and 448 children from Finland were also studied at the age of 4 years. Invitation letter to participate in the study was sent to all 3-years children living in South Estonia, whereas the Finnish cohort comprising 3year old children was recruited among children born in the Jorvi Hospital whose family was still living in the Espoo region. All participants were genotyped for HLA but no selection was based on the genotype. A study diary was given to the parents at the initial visit with the request that they should fill in information on food intake, infections, use of medications, vaccinations, allergies and environmental exposures. Information on the history of breastfeeding was collected at the first visit.

Non-fasting blood samples were collected by venipuncture from all participants at every visit. Serum was separated by centrifugation and stored in aliquots at $-70^{\circ} \mathrm{C}$ for further use. For each blood sample, $C D$ related antibody analyses were performed in accordance with a specific protocol. If the antibody test result indicated suspicion of $C D$, the child was invited for gastroduodenoscopy and small intestinal biopsy. All children with biopsy-confirmed CD were included in the current analysis. For every child with $\mathrm{CD}$, one control child was selected (except for one child in the YCC who had two controls - one at the age of 3 and another at the age of 5 years). The control children were selected from the DIABIMMUNE Study cohorts and matched for the CD-specific HLA DR/DQ genotype, country of birth, age and gender.

\subsection{Laboratory methods}

\subsubsection{HLA genotyping}

The HLA DR/DQ genotypes were analyzed with a PCR-based lanthanide labelled oligonucleotide hybridization method using time-resolved fluorometry for detection [23].

\subsubsection{Antibody analyses}

All autoantibody analyses were made at one laboratory - the Institute of Biomedicine and Translational Medicine, University of Tartu, Estonia. Immunoglobulin A tissue transglutaminase antibodies (IgA-tTG) were measured by a fully automated solid-phase fluoroenzyme immunoassay technology (ImmunoCAP EliA, Phadia $A B$, Uppsala, Sweden). According to the manufacturer, the clinical sensitivity of ImmunoCAP EliA is $96 \%$ and specificity is $99 \%$. IgAtTG values higher than $10 \mathrm{EliA} U / \mathrm{ml}$ were considered positive, and the values lower than $7 \mathrm{EliA} \mathrm{U} / \mathrm{ml}$ were considered negative. When a IgA-tTG test result was equivocal (7-10 EliA U/ml), an in-house ELISA for IgA-tTG [24] and an in-house immunofluorescence assay for IgA type endomysium autoantibodies (EMA) [25] were applied. In the case of a low serum volume, an in-house IgA-tTG ELISA with recombinant human tissue transglutaminase was used. If a serum 
sample was haemolytic, or if the IgA-tTG assay could not detect any IgA ("low RU" as a sign of possible IgA deficiency), the sera were retested for IgG-deamidated gliadin and IgG-tTG antibodies by ImmunoCAP EliA, using the same cut-off values for positivity as in the case of IgA-tTG EliA.

\subsection{Diagnosis of $C D$}

The diagnosis of CD was made in accordance with the European Society for Paediatric Gastroenterology, Hepatology and Nutrition (ESPGHAN) guidelines [1]-positive IgA-tTG antibodies and a biopsy of the small intestine with an abnormal microscopic finding. The small intestine microscopic findings were classified in accordance with the Marsh classification modified by Oberhuber [26].

\subsection{Statistical analysis}

Statistical analysis was performed with the R software for Windows (The R Foundation for Statistical Computing, Vienna, Austria). The cumulative incidence of $C D$ was calculated by dividing the number of new histologically proven $C D$ cases by the number of the study population during the study period. The incidence rate was calculated by dividing the number of new histologically proven $C D$ cases by the sum of the time each person was observed, totaled for all persons. For statistical analysis of the cumulative incidence, the Fisher's exact test or the Chi square test was applied. Comparisons between the $\mathrm{CD}$ group and the control group were performed with the Mann-Whitney-Wilcoxon test. A $P$-value $<0.05$ was considered significant.

\subsection{Ethical considerations}

The study was conducted in accordance with the ethical guidelines established by the Declaration of Helsinki and approved by local Ethics Committees for medical investigations in Estonia and in Finland. The parents of all participants provided written informed consent for the study.

\section{Results}

\subsection{General characteristics of the participants}

During the study period 29 children developed CD: nine children (two Estonian and seven Finnish children) in the BC and 20 children (six Estonian and 14 Finnish children) in the YCC. None of them had IgA deficiency and all 29 were positive for IgA-tTG and EMA. One Finnish child with CD in YCC later developed diabetes. In addition, two Finnish children in the YCC tested positive for both IgA-tTG and EMA, however, a small intestine biopsy did not confirm the diagnosis of $C D$. All children with $C D$ and those of the control group had been born full-term (gestational age between 37 and 42 weeks). Twenty-three children with CD (79\%) were autumn-, winter- or spring-born (from September to May). Five children in the CD group (17\%) and six children in the control group (20\%) had been delivered by Caesarean section while all the remaining children had been born vaginally. There were no statistical differences in mean birth weight either between the $\mathrm{CD}$ and the control groups in the $\mathrm{BC}$ ( $3632 \mathrm{~g}$ vs $3695 \mathrm{~g}$ ) or in the YCC (3520 $\mathrm{g}$ vs $3734 \mathrm{~g}$ ), or between the Estonian and Finnish children. There were more girls than boys with $C D(19$ vs $10, P=0.04)$. In the YCC there were more girls than boys with $\mathrm{CD}$ ( 15 vs five), but not in the $\mathrm{BC}$ (four vs five) $(P=0.20)$. In the CD group, 28 children carried the DR3-DQ2 haplotype (97\%) and one child in the YCC carried the DR4-DQ8 haplotype. The mean age at initial seroconversion to tTG-IgA positivity in the BC was 3.0 (95\% CI 2.5-3.6) years in Estonia and 2.3 (95\% CI 1.6-2.9) years in Finland $(P=0.12)$. In the YCC, five out of six Estonian children
Table 1

Mean duration of breastfeeding for the study subjects (BC - birth cohort, YCC young children's cohort, $\mathrm{CD}$ - coeliac disease, $N$ - number of children with available data).

\begin{tabular}{lrrlrl}
\hline & $N$ & Mean duration & $\begin{array}{l}\text { Minimum and } \\
\text { maximum }\end{array}$ & $95 \%$ CI & $P$-value \\
\hline BC CD & 9 & 35.7 weeks & $6 \ldots 96$ weeks & $13.9-57.4$ & 0.45 \\
BC control & 9 & 43.7 weeks & $1 \ldots 84$ weeks & $24.6-62.7$ & \\
YCC CD & 18 & 8.5 months & $0 \ldots 24$ months & $5.7-11.4$ & 0.12 \\
YCC control & 18 & 11.6 months & $2 \ldots 22$ months & $8.9-11.4$ & \\
\hline
\end{tabular}

(83\%) and eleven out of fourteen Finnish children (79\%) showed positive IgA-tTG at their first visit at the age of 3 years. There was no statistically significant difference in mean IgA-tTG level at the time of the diagnosis between the Estonian and Finnish children with $C D$ either in the $\mathrm{BC}(68.5 \mathrm{EliA} \mathrm{U} / \mathrm{ml}(95 \% \mathrm{CI} 0.6-08.5)$ vs $261.2 \mathrm{EliA} \mathrm{U} / \mathrm{ml}$ (95\% CI 0.6-19.1), $P=0.67)$ or in the YCC $(1045.9$ EliA U $/ \mathrm{ml}(95 \% \mathrm{CI}$ $0.27-49.9)$ vs $812.8 \mathrm{EliA} \mathrm{U} / \mathrm{ml}(95 \% \mathrm{CI} 87.8-1537.8), P=0.78)$. The IgA-tTG level of 18 out of 29 children with CD was more than 10 times as high as the cut-off level for antibody positivity $(>100$ EliA $\mathrm{U} / \mathrm{ml}$ )-in $63 \%$ of the Estonian and in $62 \%$ of the Finnish children with CD.

\subsection{Cumulative incidence}

The cumulative incidence of $\mathrm{CD}$ in the two countries was not significantly different for the $\mathrm{BC}$ or for the YCC during the study period. In the $\mathrm{BC}$ the cumulative incidence was $0.12 \%$ (95\% CI 0.02-0.49) in Estonia and $0.53 \%(95 \% \mathrm{CI} 0.23-1.13)$ in Finland $(P=0.10)$ (the incidence rate 0.4 cases per 1000 person-years and 1.8 cases per 1000 person-years, respectively), while in the YCC it was $0.4 \%$ (95\% CI $0.18-1.0$ ) and $1 \%(95 \% \mathrm{CI} 0.58-1.7)$ (the incidence rate 2.2 cases per 1000 person-years and 5.1 cases per 1000 person-years, respectively), $(P=0.12)$. However, when the cohorts were combined the cumulative incidence of childhood $C D$ was significantly higher in Finland than in Estonia ( $0.77 \%$ vs $0.27 \% ; P=0.01$ ) (the incidence rate 1.0 cases per 1000 person-years and 3.1 cases per 1000 personyears, respectively).

\subsection{Early feeding}

In the $\mathrm{BC}$ information about the duration of breastfeeding in weeks was available for all children, while in the YCC this information (in months) was available for 18 out of the 20 children (Table 1). There was no statistical difference in the total duration of breastfeeding between the children with $\mathrm{CD}$ and the control children in the BC or in the YCC (Table 1). Nor was there any significant difference in the duration of exclusive breastfeeding between the groups in the $\mathrm{BC}$. In the $\mathrm{BC}$, the age at which wheat, barley or rye was introduced was not statistically different between the $C D$ and the control groups (Table 2). The mean age when complementary food was introduced was similar in both groups (4.4 months). In the YCC, information about age at the introduction of cereal or complementary food was not collected.

\section{Table 2}

Mean age (months) of the study subjects in birth cohort at the time of cereal introduction ( $N$ - number of children with available data).

\begin{tabular}{llllll}
\hline & $\mathrm{CD}(N)$ & $95 \% \mathrm{CI}$ & Control $(N)$ & $95 \% \mathrm{CI}$ & $P$-value \\
\hline Wheat & $6.4(9)$ & $5.6-7.2$ & $5.8(8)$ & $4.9-6.7$ & 0.28 \\
Barley & $6.3(8)$ & $5.5-7.2$ & $5.6(8)$ & $4.8-6.5$ & 0.20 \\
Rye & $7.4(7)$ & $5.8-8.9$ & $5.9(8)$ & $4.7-7.1$ & 0.10 \\
\hline
\end{tabular}




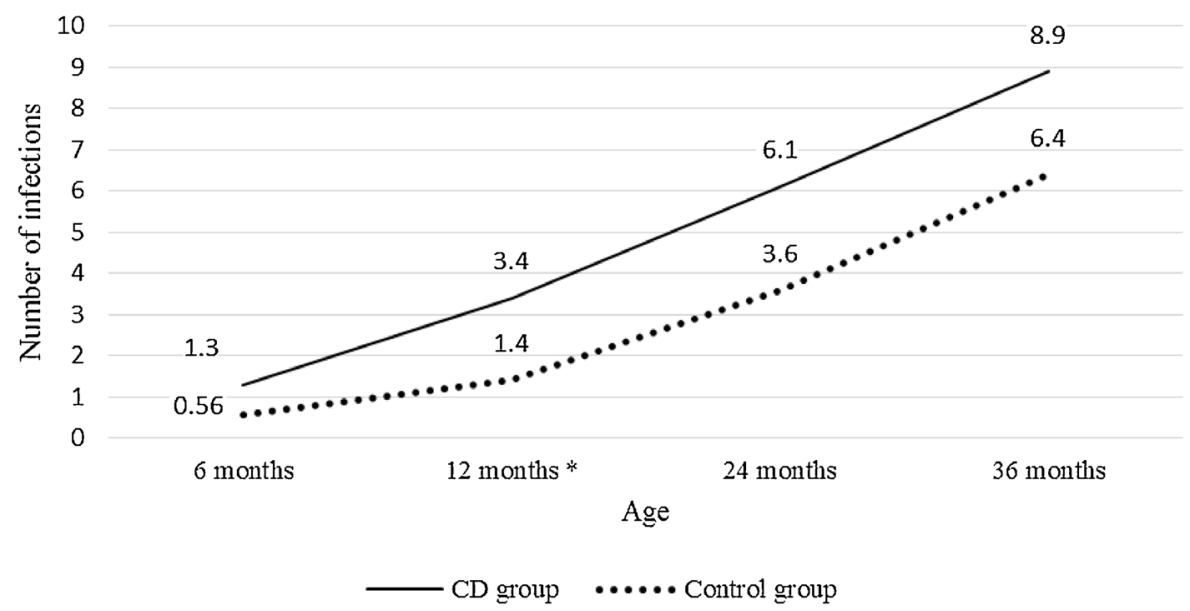

Fig. 1. Mean cumulative number of episodes of infections with fever during the study period in the birth control subjects. $\left({ }^{*}\right.$ statistically significant).

\subsection{Infections and antibiotic treatment}

In the $\mathrm{BC}$, children with $\mathrm{CD}$ had significantly more episodes of infections with fever by the age of 12 months compared to the controls ( 3.4 vs $1.4 ; P=0.04$ ). During the following months these differences remained non-significant (Fig. 1). In the BC there was no significant difference in the number of episodes of gastroenteritis between the $\mathrm{CD}$ and the control groups during the first 3 years of life ( 1 vs $0.9, P=0.82$ ). No difference was seen in the use of antibiotics between the groups, either.

In the YCC, there were no statistical differences in the number of episodes of infections with fever (2.2 vs 3.3) or antibiotic therapy (1.1 vs 1.0) between the groups. Data for the first 3 years of life was collected retrospectively. By the age of 3 years children both in the CD group and in the control group had had 3.2 episodes of otitis $(P=0.69)$ and 0.5 and 0.8 episodes of gastroenteritis, respectively $(P=0.73)$.

\subsection{Vaccinations}

In the $\mathrm{BC}$ and in the YCC, all children in both groups were at least partly vaccinated according to the local vaccination schedule, except for one Finnish control child in the BC and one Finnish child with CD and one Finnish control child in the YCC, who were not vaccinated.

\section{Discussion}

The present study compares the cumulative incidence of $\mathrm{CD}$ and a set of risk factors for Estonia and Finland, the two neighbouring countries participating in the DIABIMMUNE project. The project was initiated to test the hygiene hypothesis for T1D and for other immune-mediated diseases by using two longitudinal cohorts of children that were followed from birth to 3 years of age and from 3 to 5 years of age.

Previous studies have revealed significant unexplained differences in the prevalence of adulthood CD across Europe [4]. Some differences have been detected also in the prevalence of childhood $\mathrm{CD}$ between European countries [5,27,28]. However, the results are not well comparable as different methods have been applied and different study populations have been studied. In the present study we used the same strategy for two adjacent countries to identify new patients with $\mathrm{CD}$ and the obtained results are well comparable. Since the antibody assays for CD screening were performed in a single laboratory, the theoretical chance of revealing new cases was equal for both populations. In addition, Ress et al. [29] showed that the diagnostic accuracy and sensitivity of the fully automated fluoroenzyme immunoassay method on ImmunoCAP is similar to the traditional ELISA. Based on this approach, we found that the cumulative incidence of $C D$ in children from birth to the age of 5 was almost three times lower in Estonia than in Finland $(0.27 \%$ vs $0.77 \%$ ). A similar difference between Estonia and Finland was also noted for another immune-mediated disease, T1D [30]. The potential reasons for this may be related to different environmental factors, since differences in the risk associated genetic factors between the two populations can hardly explain the difference. The frequency of the major CD risk haplotype was actually slightly higher among the Estonian than among the Finnish population [31]. Environmental factors may start playing a role in susceptibility to immune-mediated diseases already in utero [32].

The Finnish children in the BC tended to develop positive tTG antibodies earlier compared to their Estonian peers. The IgA-tTG became positive at a mean age of 3.0 years in Estonia and at 2.3 years in Finland. Although no earlier studies have compared CDassociated autoantibodies between Estonia and Finland, it has been found that the anti-reticulin autoantibody frequency is lower for Estonian young adults compared to Swedish young adults [33], Sweden being a country with an economic and social background similar to that of Finland. The above findings support the lower prevalence of autoimmunity and autoimmune diseases in Estonia compared to the Nordic countries.

The ESPGHAN and the European Food Safety Authority recommend to avoid gluten introduction to children less than 4 months of age and more than 7 months of age and to introduce gluten while the infant is still breastfed [34,35]. According to the American Academy of Pediatrics, complementary food should be introduced between 4 and 6 months of age [36]. However, recent studies suggest that these recommendations need to be reviewed $[18,19,37]$. According to current recommendations in Estonia and Finland, complementary food should be introduced when the infant has reached the age of 6 months, although small tasting portions may be given after the age of 4 months. In our study we did not note any significant difference in the mean age at the time of cereal introduction between the $\mathrm{CD}$ and the control groups. Nor was there any difference in the total duration of breastfeeding between the children with CD and the control children either in the BC or in the YCC. These findings indicate that differences in early feeding may not be an important risk factor in modulating the development of CD.

There are more important risk factors that may be associated with differences in cereal consumption and in the quantity and 
character of ingested gluten or gluten-related prolamines. Unfortunately, we could not calculate exact cereal consumption or evaluate the quantity of gluten consumed by our study population.

Previous epidemiological studies have suggested that neonatal and early infections may increase the risk of $\operatorname{CD}[38,39]$. Myléus et al. reported that three or more infectious episodes during the first 6 months increased the risk of $C D$ later in life [38] while the type of infection was irrelevant. Also a more recent prospective large-scale population-based cohort study by Mårild et al. [40] found that early life infections may play a role in the development of $\mathrm{CD}$. Our study supports this finding. In the BC there were more infections with fever in the CD group. Similarly to a study by Myléus et al. [38], the episodes of infections were reported by the parents in our study. In the YCC the number of infections did not differ between the CD children and the controls. However, in the YCC the parents were asked to report retrospectively all their children's infections from birth up to the age of 3 years at the initial study centre visit. In the $\mathrm{BC}$, where follow-up visits were conducted with shorter intervals, the parents were asked to report infections in real time in the study diary. In contrast to Stene et al. [39], we did not find any difference in the frequency of gastroenteritis between the $\mathrm{CD}$ and the control groups. The exact molecular mechanisms by which infections increase the risk of $C D$ have not been defined. One reason could be the increased mucosal permeability of not only the intestine but also of the respiratory tract $[41,42]$. The latter circumstance may increase susceptibility to respiratory tract infections $[43,44]$. According to our data, it is not likely that differences in vaccination between the study groups and the two countries would cause differences in autoimmunity.

The major strengths of the present study are its prospective design (with part of data obtained retrospectively) and the fact that both centres in the two countries involved used identical protocols for follow-up and diagnosis. In addition, all CD antibody analyses were carried out in one laboratory and in all CD cases the diagnosis was confirmed by a biopsy of the small intestine. However, the study has also some limitations. The number of cases is relatively modest, limiting the statistical power of the study. The relatively short follow-up period (up to 3 years in the BC and from 3 to 5 years in the YCC) prevented us from detecting all childhood CD cases. Some data (early feeding and early infections in the YCC) was collected retrospectively, which may have affected its quality.

\section{Conclusion}

There is a significant difference in the 5-year cumulative incidence of childhood CD between Estonia and Finland, which is higher in the latter country. The children from Finland tended to develop IgA-tTG antibodies earlier than the children from Estonia. No difference was seen in the duration of breastfeeding or age at cereal introduction between the children with $\mathrm{CD}$ and the control children. Sequential infections early in life may increase the risk for developing $\mathrm{CD}$, although we cannot fully rule out that this was a chance finding.

\section{Conflict of interest}

None declared.

\section{Funding}

The project was funded by the European Commission (7th Framework Programme, project 202063), the Estonian Research Council grant IUT20-43 and the Academy of Finland (the Centre of Excellence in Molecular Systems Immunology and Physiology Research, 2012-2017, Decision Nos. 250114 and 284597).

\section{Acknowledgements}

The authors like to thank Ija Talja, MSc, for performing the EMA tests.

\section{Appendix A. Investigators of DIABIMMUNE Study Group}

Mikael Knip, Children's Hospital, University of Helsinki, Finland Katriina Koski, Institute of Clinical Medicine, University of Helsinki, Finland

Matti Koski, Institute of Clinical Medicine, University of Helsinki, Finland

Taina Härkönen, Children's Hospital, University of Helsinki, Finland Heli Siljander, Children's Hospital, University of Helsinki, Finland Samppa Ryhänen, Children's Hospital, University of Helsinki, Finland

Anu-Maaria Hämäläinen, Jorvi Hospital, Helsinki University Central Hospital, Finland

Anne Ormisson, Children's Clinic, Tartu University Hospital, Finland Aleksandr Peet, Department of Pediatrics, Tartu University Hospital, Finland

Vallo Tillmann, Department of Pediatrics, Tartu University Hospital, Finland

Valentina Ulich, Ministry of Health and Social Development, Karelian Republic of the Russian Federation, Russia

Elena Kuzmicheva, Ministry of Health and Social Development, Karelian Republic of the Russian Federation, Russia

Sergei Mokurov, Ministry of Health and Social Development, Karelian Republic of the Russian Federation, Russia

Svetlana Markova, Children's Republic Hospital, Karelian Republic of the Russian Federation, Russia

Svetlana Pylova, Children's Republic Hospital, Karelian Republic of the Russian Federation, Russia

Marina Isakova, Perinatal Center, Karelian Republic of the Russian Federation, Russia

Elena Shakurova, Perinatal Center, Karelian Republic of the Russian Federation, Russia

Vladimir Petrov, Maternity Hospital N8 1, Petrozavodsk, Russia Natalya V. Dorshakova, Petrozavodsk State University, Russia

Tatyana Karapetyan, Petrozavodsk State University, Russia

Tatyana Varlamova, Petrozavodsk State University, Russia

Jorma Ilonen, Immunogenetics Laboratory, University of Turku, Finland

Department of Clinical Microbiology, University of Eastern Finland, Kuopio, Finland

Minna Kiviniemi, Immunogenetics Laboratory, University of Turku, Finland

Kristi Alnek, Department of Immunology, University of Tartu, Finland

Helis Janson, Department of Immunology, University of Tartu, Finland

Raivo Uibo, Department of Immunology, University of Tartu, Finland

Erika von Mutius, Children's Hospital, Ludwig Maximilians Universität, Munchen, Germany

Juliane Weber, Children's Hospital, Ludwig Maximilians Universität, Munchen, Germany

Helena Ahlfors, Turku Centre of Biotechnology, University of Turku and Åbo Akademi, Finland

Henna Kallionpää, Turku Centre of Biotechnology, University of Turku and Åbo Akademi, Finland

Essi Laajala, Turku Centre of Biotechnology, University of Turku and Åbo Akademi, Finland

Riitta Lahesmaa, Turku Centre of Biotechnology, University of Turku and Åbo Akademi, Finland 
Harri Lähdesmäki, Turku Centre of Biotechnology, University of Turku and Åbo Akademi, Finland

Robert Moulder, Turku Centre of Biotechnology, University of Turku and Åbo Akademi, Finland

Janne Nieminen, Department of Vaccination and Immune Protection, National Institute for Health and Welfare, Helsinki, Finland Terhi Ruohtula, Department of Vaccination and Immune Protection, National Institute for Health and Welfare, Helsinki, Finland Outi VaaralaDepartment of Vaccination and Immune Protection, National Institute for Health and Welfare, Helsinki, Finland Hanna Honkanen, Department of Virology, University of Tampere and Tampere University Hospital, Finland

Heikki Hyöty, Department of Virology, University of Tampere and Tampere University Hospital, Finland

Anita Kondrashova, Department of Virology, University of Tampere and Tampere University Hospital, Finland

Sami Oikarinen, Department of Virology, University of Tampere and Tampere University Hospital, Finland

Hermie J.M. Harmsen, University Medical Center Groningen, Finland

Marcus C. De Goffau, University Medical Center Groningen, Finland Gjal Welling, University Medical Center Groningen, Finland Kirsi Alahuhta, Department for Welfare and Health Promotion, National Institute for Health and Welfare, Helsinki, Finland Suvi M. Virtanen, Department for Welfare and Health Promotion, National Institute for Health and Welfare, Helsinki, Finland

\section{References}

[1] Husby S, Koletzko S, Korponay-Szabó IR, et al. European Society for Pediatric Gastroenterology, Hepatology, and Nutrition guidelines for the diagnosis of coeliac disease. Journal of Pediatric Gastroenterology and Nutrition 2012;54:136-60

[2] Kang J, Kang A, Green A, et al. Systematic review: worldwide variation in the frequency of coeliac disease and changes over time. Alimentary Pharmacology and Therapeutics 2013;38:226-45.

[3] Rubio-Tapia A, Ludvigsson JF, Brantner TL, et al. The prevalence of celiac disease in the United States. American Journal of Gastroenterology 2012;107:1538-44.

[4] Mustalahti K, Catassi C, Reunanen A, et al. The prevalence of celiac disease in Europe: results of a centralized, international mass screening project. Annals of Medicine 2010;42:587-95.

[5] Myléus A, Ivarsson A, Webb C, et al. Celiac disease revealed in 3\% of Swedish 12 -year-olds born during an epidemic. Journal of Pediatric Gastroenterology and Nutrition 2009;49:170-6.

[6] Ress K, Luts K, Rägo T, et al. Nationwide study of childhood celiac disease incidence over a 35-year period in Estonia. European Journal of Pediatrics 2012;171:1823-8.

[7] Zingone F, West J, Auricchio R, et al. Incidence and distribution of coeliac disease in Campania (Italy): 201--2013. United European Gastroenterology Journal 2015;3:182-9.

[8] Zingone F, West J, Crooks CJ, et al. Socioeconomic variation in the incidence of childhood coeliac disease in the UK. Archives of Disease in Childhood 2015;100:466-73.

[9] Lohi S, Mustalahti K, Kaukinen K, et al. Increasing prevalence of coeliac disease over time. Alimentary Pharmacology and Therapeutics 2007;26:1217-25.

[10] Kagnoff MF. Celiac disease (A gastrointestinal disease with environmental, genetic, and immunologic components). Gastroenterology Clinics of North America 1992;21:405-25.

[11] Karell K, Louka AS, Moodie SJ, et al. HLA types in celiac disease patients not carrying the DQA $1 * 05-\mathrm{DQB} 1 * 02(\mathrm{DQ} 2)$ heterodimer: results from the European Genetics Cluster on Celiac Disease. Human Immunology 2003;64:469-77.

[12] Hadithi M, von Blomberg BM, Crusius JB, et al. Accuracy of serologic tests and HLA-DQ typing for diagnosing celiac disease. Annals of Internal Medicine 2007:147:294-302.

[13] Fälth-Magnusson K, Franzen L, Jansson G, et al. Infant feeding history shows distinct differences between Swedish celiac and reference children. Pediatric Allergy and Immunology 1996;7:1-5.

[14] Ivarsson A, Hernell O, Stenlund $\mathrm{H}$, et al. Breast-feeding protects against celiac disease. American Journal of Clinical Nutrition 2002;75:914-21.

[15] Akobeng AK, Ramanan AV, Buchan I, et al. Effect of breast feeding on risk of coeliac disease: a systematic review and meta-analysis of observational studies. Archives of Disease in Childhood 2006;91:39-43.

[16] Peters U, Schneeweiss S, Trautwein EA, et al. A case-control study of the effect of infant feeding on celiac disease. Annals of Nutrition and Metabolism 2001;45:135-42.

[17] Norris JM, Barriga K, Hoffenberg EJ, et al. Risk of celiac disease autoimmunity and timing of gluten introduction in the diet of infants at increased risk of disease. Journal of the American Medical Association 2005;293: 2343-51.

[18] Vriezinga SL, Auricchio R, Bravi E, et al. Randomized feeding intervention in infants at high risk for celiac disease. New England Journal of Medicine 2014;371:1304-15.

[19] Lionetti E, Castellaneta S, Francavilla R, et al. Introduction of gluten, HLA status, and the risk of celiac disease in children. New England Journal of Medicine 2014;371:1295-303.

[20] Lewy H, Meirson H, Laron Z. Seasonality of birth month of children with celiac disease differs from that in the general population and between sexes and is linked to family history and environmental factors. Journal of Pediatric Gastroenterology and Nutrition 2009;48:181-5.

[21] Rostami Nejad M, Ishaq S, Al Dulaimi D, et al. The role of infectious mediators and gut microbiome in the pathogenesis of celiac disease. Archives of Iranian Medicine 2015;18:244-9.

[22] Peet A, Kool P, Ilonen J, et al. Birth weight in newborn infants with different diabetes-associated HLA genotypes in three neighbouring countries: Finland, Estonia and Russian Karelia. Diabetes/Metabolism Research and Reviews 2012;28:455-61

[23] Hermann R, Turpeinen H, Laine AP, et al. HLA DR-DQ-encoded genetic determinants of childhood-onset type 1 diabetes in Finland: an analysis of 622 nuclear families. Tissue Antigens 2003;62:162-9.

[24] Teesalu K, Agardh D, Panarina M, et al. A modified ELISA for improved detection of IgA, IgG, and IgM anti-tissue transglutaminase antibodies in celiac disease. Clinica Chimica Acta 2009;403:37-41.

[25] Uibo O, Teesalu K, Metsküla K, et al. Screening for celiac disease in Down's syndrome patients revealed cases of subtotal villous atrophy without for celiac disease HLA-DQ and tissue transglutaminase antibodies. World Journal of Gastroenterology 2006;12:1430-4.

[26] Oberhuber G, Granditsch G, Vogelsang H. The histopathology of coeliac disease: time for a standardized report scheme for pathologists. European Journal of Gastroenterology and Hepatology 1999;11:1185-94.

[27] Kondrashova A, Mustalahti K, Kaukinen K, et al. Lower economic status and inferior hygienic environment may protect against celiac disease. Annals of Medicine 2008;40:223-31.

[28] Laass MW, Schmitz R, Uhlig HH, et al. The prevalence of celiac disease in children and adolescents in Germany. Deutsches Ärzteblatt International 2015;112:553-60.

[29] Ress K, Harro J, Uibo O, et al. Use of a fully automated immunoassay for celiac disease screening in a pediatric population. Clinical Chemistry and Laboratory Medicine 2011;49:983-7.

[30] DIAMOND Project Group. Incidence and trends of childhood type 1 diabetes worldwide 1990-1999. Diabetic Medicine 2006;23:857-66

[31] Nejentsev S, Koskinen S, Sjöroos M, et al. Distribution of insulin-dependent diabetes mellitus (IDDM)-related HLA alleles correlates with the difference in IDDM incidence in four populations of the Eastern Baltic region. Tissue Antigens 1998;52:473-7.

[32] Kallionpää H, Laajala E, Öling V, et al. Standard of hygiene and immune adaptation in newborn infants. Clinical Immunology 2014;155:136-47.

[33] Uibo R, Talja I, Jõgi T, et al. Autoantibodies in Estonia and Sweden, populations with different response to allergens. International Archives of Allergy and Immunology 1998;117:126-30.

[34] Agostoni C, Decsi T, Fewtrell M, et al. Complementary feeding: a commentary by the ESPGHAN Committee on Nutrition. Journal of Pediatric Gastroenterology and Nutrition 2008;46:99-110.

[35] EFSA Panel on Dietetic Products, Nutrition and Allergies (NDA). Scientific opinion on the appropriateage for introduction of complementary feeding of infants; 2015. Available at: http://www.efsa.europa.eu/en/efsajournal/doc/ 1423.pdf.

[36] Kleinman RE, editor. Pediatric nutrition handbook. 6th ed. Elk Grove Village, IL: American Academy of Pediatrics; 2009.

[37] Szajewska H, Shamir R, Chmielewska A, et al. Systematic review with metaanalysis: early infant feeding and coeliac disease - update 2015. Alimentary Pharmacology and Therapeutics 2015;41:1038-54

[38] Myléus A, Hernell O, Gothefors L, et al. Early infections are associated with increased risk for celiac disease: an incident case-referent study. BMC Pediatrics 2012:12:194-201.

[39] Stene LC, Honeyman MC, Hoffenberg EJ, et al. Rotavirus infection frequency and risk of celiac disease autoimmunity in early childhood: a longitudinal study. American Journal of Gastroenterology 2006;101:2333-40.

[40] Mårild K, Kahrs CR, Tapia G, et al. Infections and risk of celiac disease in childhood: a prospective nationwide cohort study. American Journal of Gastroenterology 2015;110:1475-84.

[41] Sapone A, Lammers KM, Casolaro V, et al. Divergence of gut permeability and mucosal immune gene expression in two gluten-associated conditions: celiac disease and gluten sensitivity. BMC Medicine 2011;9: 23-33.

[42] Robertson DA, Taylor N, Sidhu H, et al. Pulmonary permeability in coeliac disease and inflammatory bowel disease. Digestion 1989;42:98-103.

[43] Mårild K, Fredlund H, Ludvigsson JF. Increased risk of hospital admission for influenza in patients with celiac disease: a nationwide cohort study in Sweden. American Journal of Gastroenterology 2010;105:2465-73.

[44] Tjernberg AR, Ludvigsson JF. Children with celiac disease are more likely to have attended hospital for prior respiratory syncytial virus infection. Digestive Diseases and Sciences 2014;59:1502-8. 